\title{
Optimization of FDG PET study after mRNA COVID-19 vaccination to reduce the interference of vaccine-associated hypermetabolic lymphadenopathy
}

\author{
Wajana Thaweerat ${ }^{1}$ (D)
}

Received: 4 December 2021 / Accepted: 13 December 2021 / Published online: 30 January 2022

(c) The Author(s) under exclusive licence to The Japanese Society of Nuclear Medicine 2021

Messenger RNA (mRNA) COVID-19 vaccine is one of the main vaccine types currently used for preventing SARS$\mathrm{CoV}-2$ infection worldwide. However, its administration can interfere with an FDG PET study since an increased uptake of 18F-FDG can be observed at the vaccine injection site and adjacent regional lymph nodes. The interpretation of PET study in oncologic patients might be impacted especially in head and neck cancer, breast cancer or lymphoma in which supraclavicular and axillary lymph node metastases are suspected. Hence, Advani et al. [1] suggest that FDG PET study should be performed at least 2 weeks after vaccination to reduce the interference of lymphadenopathy causing by vaccination in PET interpretation.

Nevertheless, the onset and duration of hypermetabolic lymphadenopathy are different in each received dose which might assist in the optimization of the timing of PET scan. A large Israeli cohort study reported differences in the frequency and intensity of vaccine-associated hypermetabolic lymphadenopathy (VAHL) between the first and second doses in which higher frequency and intensity were observed following the second dose [2]. Three window periods comprising the first 5 days after the first dose, the third week after the first dose or before the second dose, and at least 3 weeks after the second dose were suggested for performing PET study due to the lower incidence of VAHL of 7.4\%, $24.7 \%$, and $29.0 \%$, respectively [2]. Another Israeli cohort study reported that VAHL at the ipsilateral axillary region can still be observed in $29 \%$ of patients during 7-10 weeks after receiving the second dose of mRNA COVID vaccine [3]. Therefore, scheduling PET just before the second dose of vaccination might be the best option to prolong the

Wajana Thaweerat

wajana.tha@gmail.com

1 Division of Radiation Oncology, Department of Radiology, Faculty of Medicine Siriraj Hospital, Mahidol University, Bangkok, Thailand duration after the first dose and avoid the greater effect of vaccine-associated hypermetabolic lymphadenopathy from the second dose.

For patients receiving the third booster dose of mRNA vaccine, the incidence of VAHL at the axillary and supraclavicular regions during 11-15 days and at least 16 days from the booster dose were $17.7 \%$ and $2.8 \%$, respectively [4]. Thus, performing an FDG PET study more than 2 weeks after vaccine administration as suggested by Advani et al. [1] might be applicable to oncologic patients who receive the third booster shot since the incidence of VAHL was low during the recommended period.

The selection of the vaccine injection site could also assist in PET study interpretation. The scientific expert panel recommended that the vaccine be administered at the contralateral arm to the primary tumor site [5]. Furthermore, anterolateral thigh which is another approved vaccination site might be recommended for patients who had some malignancy at the head and neck, thorax, or upper extremities to prevent VAHL from occurring at the respective regional lymph nodes. Nonetheless, VAHL still needs further investigation before establishing an evidence-based guideline on the optimization of timing between vaccination and PET study to reduce VAHL which may interfere with PET result interpretation.

\section{References}

1. Advani P, Chumsri S, Pai T, Li Z, Sharma A, Parent E. Temporal metabolic response to mRNA COVID-19 vaccinations in oncology patients. Ann Nucl Med. 2021;35(11):1264-9. https://doi.org/ 10.1007/s12149-021-01675-8.

2. Cohen D, Krauthammer SH, Wolf I, Even-Sapir E. Hypermetabolic lymphadenopathy following administration of BNT162b2 mRNA Covid-19 vaccine: incidence assessed by [18F]FDG PET-CT and relevance to study interpretation. Eur J Nucl Med 
Mol Imaging. 2021;48(6):1854-63. https://doi.org/10.1007/ s00259-021-05314-2.

3. Eshet Y, Tau N, Alhoubani Y, Kanana N, Domachevsky L, Eifer M. Prevalence of increased FDG PET/CT axillary lymph node uptake beyond 6 weeks after mRNA COVID-19 vaccination. Radiology. 2021;300(3):E345-7. https://doi.org/10.1148/radiol. 2021210886.

4. Cohen D, Hazut Krauthammer S, Wolf I, Even-Sapir E. A sigh of relief: vaccine-associated hypermetabolic lymphadenopathy following the third COVID-19 vaccine dose is short in duration and uncommonly interferes with the interpretation of $[18 \mathrm{~F}]$ FDG PET-CT studies performed in oncologic patients. Eur J Nucl Med Mol Imaging. 2021;15:1-7. https://doi.org/10.1007/ s00259-021-05579-7.
5. Becker AS, Perez-Johnston R, Chikarmane SA, Chen MM, El Homsi M, Feigin KN, Gallagher KM, Hanna EY, Hicks M, Ilica AT, Mayer EL, Shinagare AB, Yeh R, Mayerhoefer ME, Hricak H, Vargas HA. Multidisciplinary recommendations regarding postvaccine adenopathy and radiologic imaging: radiology scientific expert panel. Radiology. 2021;300(2):E323-7. https://doi.org/10. 1148/radiol.2021210436.

Publisher's Note Springer Nature remains neutral with regard to jurisdictional claims in published maps and institutional affiliations. 